\title{
Six critical questions for DNA repair enzymes in skincare products: a review in dialog
}

This article was published in the following Dove Press journal:

Clinical, Cosmetic and Investigational Dermatology

\author{
Daniel B Yarosh' \\ Amanda Rosenthal ${ }^{2,3}$ \\ Ronald Moy ${ }^{3}$ \\ 'Daniel B Yarosh Inc., Merrick, NY, USA; \\ ${ }^{2}$ Department of Dermatology and \\ Cutaneous Surgery, University of Miami, \\ Miami, FL, USA; ${ }^{3}$ Research Department, \\ Moy-Fincher-Chipps Facial Plastics/ \\ Dermatology, Beverly Hills, CA, USA
}

\begin{abstract}
Concerns over existing sunscreen filters have reinforced the need to examine supplemental sun protection or repair of sun damage. Technology to enhance DNA repair has been available in skincare and sunscreen products for several decades, but skepticism and lack of familiarity with the supporting data remain prevalent. Here, we address six of the main questions raised by medical professionals regarding the efficacy of DNA repair enzymes in sun protection. These include the mode of delivery and mechanism of action, the effect on cellular responses and the amelioration of pre-cancers, cancers and photoaging. The conclusions are that topical DNA repair enzymes do enhance removal of DNA damage and reduce the appearance of new actinic keratoses as well as increase regression of existing lesions. Support for prevention of photoaging and skin cancer is significant but could be strengthened or disproven with additional research.
\end{abstract}

Keywords: DNA repair, skin cancer, T4 endonuclease V, photolyase, UV endonuclease, actinic keratosis

\section{Introduction}

Despite decades of sun protection warnings, the rates of both melanoma and nonmelanoma skin cancer in the US have been steadily rising, ${ }^{1}$ and the consumer concern for signs of premature aging continues unabated. Our primary topical product defense has been sunscreen use, but these are now under attack because they may damage the environment of the coral reefs, ${ }^{2}$ they may enter the bloodstream $^{3}$ and newer sunscreen chemicals have not received FDA approval due to insufficient safety support. ${ }^{4}$ We need to consider alternate technologies that can either attenuate sun exposure or reduce its biological consequences. This emphasizes the importance of prevention in an overall program of sun protection.

One such technology is the use of DNA repair enzymes to speed the removal of DNA damage from sunlight and other sources of genotoxicity. The first patent in this field was awarded in $1991 .^{5}$ Topical products with DNA repair technology have been available in the commercial market since then (Supplementary material), and the technical data supporting their efficacy have been periodically reviewed. ${ }^{6-10}$ Despite this, there remains skepticism about this approach to sun protection and repair of sun damage. Our experience is that dermatologists and other interested professionals have questions that have not been adequately addressed. This review is designed to directly address these critical issues.

\section{Methods}

The questions in this review were selected by the authors after listening to questions from colleagues, physicians, patients, and consumers posed publicly and in private
Correspondence: Daniel B Yarosh Daniel B Yarosh Inc., 2915 Shore Drive, Merrick, NY I I566, USA

Tel +l 5165107100

Email dyarosh@danyarosh.com 
over 20 years of technology commercialization. They were finalized after discussions among the authors. A search of PubMed yielded 47 papers for the search term "DNA repair liposomes AND skin" and 125 papers for the search term "DNA repair liposomes". These papers were reviewed as well as the references cited by the prior reviews (refs. 6-10).

\section{Question I: do DNA repair enzymes really work?}

The 2015 Nobel Prize in Chemistry was awarded to three scientists for mechanistic studies of DNA repair, culminating many decades of research showing DNA is able to repair itself. The surprising finding that bacterial DNA repair enzymes can indeed function inside human cells was first demonstrated nearly 45 years ago by Tanaka and colleagues. ${ }^{11}$ They permeabilized UV-irradiated human cells in culture and added a DNA repair enzyme from bacteria to increase the removal of DNA lesions and DNA repair synthesis.

The enzyme they used, T4 endonuclease V, specifically recognizes one class of UV-induced DNA damage, called cyclobutane pyrimidine dimers (CPD) and repairs DNA by catalyzing two reactions: the first uses glycosylase, which releases thymine and causes an apurinic site; the second involves AP lyase, which incises the phosphodiester backbone at the site of the missing base, causing a singlestranded break. The host cell supplies the exonuclease that then removes bases around this site, and a polymerase fills the gap, thereby repairing the photodamaged DNA. ${ }^{12}$ A similar enzyme UV endonuclease from the bacteria Micrococcus luteus also stimulates the excision repair process when introduced into mammalian cells. ${ }^{13}$

Shortly thereafter, in 1980, a completely different bacterial DNA repair enzyme, the photoreactivating enzyme, was inserted into mammalian cells and proved to be active in reversing lesions. ${ }^{14}$ This enzyme utilizes energy from blue light to rapidly repair damaged DNA by catalyzing a reaction that transfers electrons leading to the splitting of the cyclobutane ring in the damaged DNA but no strand break intermediates. ${ }^{15}$ Photolyases are found throughout the bacterial and plant kingdoms, but mammalian cells do not have their own photoreactivating enzyme; the homologous human gene has been hijacked by evolution and put to use detecting blue light to maintain the circadian rhythm. ${ }^{16}$

A third class of DNA repair enzymes has been used to enhance normal DNA repair. Here, the 8-oxoguanineDNA glycosylase 1 (OGG1) from a mustard plant
Arabidopsis thaliana was encapsulated in liposomes and used to repair oxidative damage to the guanine base in DNA. ${ }^{17}$

The finding that at least three different enzymes delivered in several different ways stimulated the removal of DNA damage should leave little doubt that exogenous bacterial and plant enzymes can and do work in mammalian and human cells.

\section{Question 2: do DNA repair enzymes actually get into skin and repair DNA?}

The best characterized DNA repair topical skincare products contain enzymes that are encapsulated in liposomes, which are multilayered lipid vesicles of about $150 \mathrm{~nm}$ diameter, composed of phospholipids that are similar to the keratinocyte cell membrane. ${ }^{18}$ After topical application, they localize to the epidermis within $1 \mathrm{hr}^{19}$ The liposomes are $\mathrm{pH}$ sensitive, so that when they are taken up by keratinocytes into the acidic lysosomal sac, the liposome membrane dissolves and the enzymes are released into the cell, where they diffuse into the nucleus and remain bound to DNA. ${ }^{19}$ Human skin was treated with $\mathrm{T} 4$ endonuclease $\mathrm{V}$ liposomes, probed with antibodies specific for the prokaryotic enzyme, and electron micrographs revealed the enzyme inside Langerhans cells and nuclei in the basal layer of epidermis. ${ }^{20}$

The precise pathway(s) by which the liposomes traverse the stratum corneum and travel down the depth of the epidermis is not known. Also unknown is the fraction of the applied enzyme that actually reaches the living tissue.

Despite these gaps in our knowledge, UV-irradiated human skin treated with DNA repair enzymes encapsulated in liposomes have significantly reduced DNA damage compared to controls in clinical studies from several research groups.

- T4 endonuclease $\mathrm{V}$ applied to patients with the genetic disease xeroderma pigmentosum (XP, enzymatic defect in DNA repair) reduced CPDs by $20 \%$ in skin biopsies ${ }^{21}$ In this study, 12 XP patients were exposed to UV at a small spot on the buttocks, the liposomal T4 endonuclease V was applied, and after $6 \mathrm{hrs}$, the spot was removed along with a control site and analyzed for CPD by immunohistochemistry.

- UV endonuclease applied to UV-irradiated normal volunteers reduced CPDs by $18 \%{ }^{22}$ Liposomal UV endonuclease was applied to a spot on the buttocks of 
9 normal volunteers, daily for 4 days. The sites were then UV irradiated, and after $1 \mathrm{hr}$, biopsies were taken and CPDs were measured by immunohistochemistry.

- Photolyase applied to the UVB-irradiated volunteers and subsequently exposed to photoreactivating light decreased the number of CPDs by 40 $45 \% .{ }^{23}$ Seven volunteers were UV irradiated and photolyase in liposomes was applied to the site. After $1 \mathrm{hr}$, the sites were exposed to photoreactivating light, and then, biopsies were immediately taken and analyzed for CPD content by immunofluorescence.

- Photolyase applied in a sunscreen formula followed by UV over a week reduced CPDs by $93 \%$ compared to a $62 \%$ reduction by sunscreen alone. ${ }^{24}$ Ten volunteers were treated with either sunscreen or sunscreen with liposomal photolyase on the lower back and then after 30 mins were UV irradiated on the site, for 4 consecutive days. Three days later, biopsies were taken from the treated sites and analyzed for CPD by ELISA.

- UV endonuclease combined with photolyase in a sunscreen used by volunteers for 6 months decreased CPDs by $61 \%$, compared to $35 \%$ with sunscreen alone. ${ }^{25}$ Twenty-eight patients with clinically evident actinic keratosis (AK) were treated on those sites with either sunscreen or sunscreen plus UV endonuclease and photolyase, twice daily for 6 months. Biopsies were taken of treated sites and CPD analyzed by ELISA.

- UV endonuclease, photolyase and OGG1 added to a sunscreen and applied by volunteers and UV irradiated over 8 days reduced CPDs by $12 \%$ and 8 oxoguanine by $17 \%$ compared to sunscreen alone. ${ }^{26}$ Twenty healthy volunteers were treated on several sites on the lower back with various formulations, including sunscreen and sunscreen plus liposomal UV endonuclease plus photolyase plus OGG1, followed by UV irradiation, for 8 consecutive days. Twenty-four hours after the last exposure, biopsies were taken and CPD and 8-oxoguanine were measured by ELISA. This study included 3 different commercial DNA repair products, all of which reduced DNA damage compared to sunscreen controls.

These robust findings using a variety of enzymes and protocols are best explained if the DNA repair enzymes penetrated into the epidermis, reached the damaged nuclei of the cells and increased the speed of removal of DNA damage.

\section{Question 3: how much skin damage can these enzymes repair?}

UV induces DNA damage that is converted to mutations which subsequently, many years later, yield pre-cancer and skin cancer. In the short run, UV-induced DNA damage immediately activates skin responses that mobilize cellular and immune defenses. These responses have been studied using a variety of molecular markers of gene and protein expression following treatment with liposomal DNA repair enzymes.

The first set of responses are considered molecular markers of UV exposure that are relevant to oncogenesis and photoaging:

- p53 gene expression is activated after UV irradiation and in chronically sun exposed skin, but in 35 patients with AK who used either sunscreen or photolyase plus sunscreen for 1 year, those who used the DNA repair enzyme had reduced expression of p53 by immunostaining. ${ }^{27}$ One consequence of elevated p53 gene expression is cell death by apoptosis in DNA damaged cells; photolyase added to a sunscreen reduced the number of apoptotic cells by $82 \%$, compared to a $40 \%$ reduction by sunscreen alone, in biopsies from 10 volunteers treated for 4 consecutive days, as measured by ELISA. ${ }^{24}$

- c-FOS is another proto-oncogene elevated following UV exposure; treatment of 12 healthy volunteers on 4 consecutive days with sunscreen plus photolyase, followed by UV and in some cases additional application of liposomal UV endonuclease, significantly reduced c-FOS upregulation measured by RT-PCR compared to sunscreen alone. ${ }^{28}$ The combination of photolyase in sunscreen before irradiation and UV endonuclease afterward almost completely abolished c-FOS expression above baseline. This study also found a reduction in UV-induced telomere shortening by DNA repair enzymes.

- Matrix Metalloproteinase I (MMP-1) is a UV inducible collagenase that contributes to photoaging. Sixteen volunteers were treated with liposomal photolyase followed by UV and photoreactivating light, then MMP-1 mRNA induction was measured by RT-PCR and protein expression was confirmed by immunohistochemistry. ${ }^{29}$ 
Photolyase reduced MMP-1 mRNA expression by $90 \%$ and protein expression in nearly all the subjects.

The next set of responses are induction of cytokines and subsequent cell responses that modulate the skin immune system. They are responsible for the UV-induced suppression of the immune system that allows the outgrowth of pre-cancers and cancers.

- Cytokines TNF $\alpha$ and IL-10 are elevated after UV exposure and contribute to the pathway of immunosuppression. Fifteen volunteers were UV irradiated on their buttocks and then treated with liposomal T4 endonuclease $\mathrm{V}$ or a heat-inactivated control lotion repeatedly, and then at $6 \mathrm{hrs}$ a biopsy was taken. ${ }^{20}$ Levels of TNF $\alpha$ and IL-10 mRNA, measured by in situ hybridization, were upregulated by UV alone and strongly downregulated in the UV + DNA repair enzyme samples. IL-10 protein, measured by immunohistochemistry, was also strongly downregulated throughout the epidermis in the treated skin.

- Sunburn, the erythema following a sufficient UV exposure, was not prevented by $\mathrm{T} 4$ endonuclease $\mathrm{V}$ treatment. $^{20,21,30}$ However, when UV-exposed skin was immediately treated with photolyase in liposome and photoreactivating light, erythema and sunburn cell formation were prevented. ${ }^{23}$ This suggests that the signals for sunburn are induced very quickly after DNA damage forms, and while immediate repair by photoreactivation can block their release, slower repair by endonuclease-initiated excision repair does not.

- Suppression of contact hypersensitivity (CHS) develops in the hours and days after UV exposure. Fifteen nickel-sensitive volunteers were UV irradiated on 4 consecutive days, and then some areas were treated with T4 endonuclease $\mathrm{V}$ liposomes and other sites left untreated; all sites were challenged with nickel patches. $^{30}$ The untreated sites failed to develop nickel-induced erythema, while the contact sensitivity reaction was $50 \%$ restored in the sites treated with DNA repair enzymes. In this study, DNA repair enzymes completely restored the UV-induced loss of CD11c+ dendritic, antigen-presenting cells from the skin, which likely accounts for the recovery of the sensitization response. Similar results were obtained in a larger study of 80 volunteers using dinitrochlorobenzene as the antigen ${ }^{22}$ where
UV-induced CHS suppression was reduced $19 \%$ with DNA repair enzymes. These findings were then repeated in a placebo-controlled study of 20 subjects using a commercial skincare product containing liposomal DNA repair enzymes. ${ }^{31}$

- Polymorphic light eruption (PLE) is a common photodermatosis with a pathogenic resistance to UV-induced immune suppression and expresses as itchy polymorphic skin lesions following UVA and UVB. Fourteen PLE patients were exposed on 4 consecutive days to suberythemal doses of solarsimulating light, followed by treatment with either a lotion containing photolyase and UV endonuclease in liposomes, or placebo, and then exposed to photoreactivating light. ${ }^{32}$ At the time of maximum PLE expression, DNA repair treated sites had $61 \%$ fewer symptoms compared to $27 \%$ for placebo sites.

In the hours after DNA damage formation in human skin, cellular alarm responses, such as phosphorylation of transcription factors by damage-responsive kinases, induce gene expression leading to release of signaling molecules that modulate the immune system. The repeated rounds of DNA damage followed by cellular distress reactions over the course of a lifetime set the stage for photoaging and skin cancer. These studies demonstrate that each of these cycles in the short term can be substantially dampened by DNA repair enzymes that lessen the initiating damage burden.

\section{Question 4: do DNA repair enzymes prevent skin cancer?}

Prospective, intervention studies of skin cancer in humans are difficult to do because of the study size and time required and ethical considerations. However, studies in mice have repeatedly demonstrated a reduction in UVinduced skin cancer after treatment with DNA repair enzymes. In the first studies, UV-irradiated mice treated with $\mathrm{T} 4$ endonuclease $\mathrm{V}$ in liposomes had a dose-dependent decrease in the incidence of squamous cell carcinoma compared to controls. ${ }^{33}$ This finding was reproduced by a second laboratory. ${ }^{34}$ A genetically modified form of $\mathrm{T} 4$ endonuclease $\mathrm{V}$ was also demonstrated to reduce $\mathrm{UV}$ induced skin cancers in mice. ${ }^{35}$ Interestingly, topical treatment of UV-irradiated mice with OGG1, which repairs oxidative damage to DNA bases, did not reduce the number of skin tumors, but did reduce tumor size and 
progression. ${ }^{36}$ This suggests that oxidative DNA damage is more important in tumor progression than initiation and demonstrates that different types of DNA damage have different impacts on carcinogenesis.

The first studies in humans were performed in XP patients with greatly elevated incidences of skin cancer, particularly on sun-exposed skin. After 1 year of topical application of $\mathrm{T} 4$ endonuclease $\mathrm{V}$ to $20 \mathrm{XP}$ patients, the appearance of new AKs was reduced by $68 \%$ and new basal cell carcinomas were reduced by $30 \%{ }^{37}$ These studies have been extended to non-XP patients using other DNA repair enzyme formulations, usually by assessing AKs.

- UV endonuclease in liposomes applied to 17 patients with existing AKs over 48 weeks resulted in a statistically significant reduction in their number. ${ }^{38}$ Application over just 8 weeks to 13 patients with AKs on their faces or scalps showed a reduction in the number of lesions, and, in the 12 weeks after treatment discontinuation, the number of AKs continued to fall in the treated group, while the number of AKs increased in the placebo group. ${ }^{39}$

- Photolyase in liposomes mixed with sunscreen and applied to patients with AKs has been studied for a decade, and 11 studies covering 228 patients have been reviewed [see ref. ${ }^{40}$ for a list of studies and their descriptions]. These studies have consistently found a reduction in the appearance of new AKs and a reduction in the number of existing lesions. For example, a study of 8 XP patients treated for 12 months with a high protection sunscreen and photolyase showed a $65 \%$ reduction in the appearance of new $\mathrm{AKs}$ and a $56 \%$ reduction in new basal cell carcinomas. ${ }^{41}$ In a series of 6 clinical cases, improvement in existing $\mathrm{AK}$ lesion count was observed within 3 months, ${ }^{42}$ and in another 9 patients, all achieved a 50\% reduction in $\mathrm{AK}$ counts within 3 months. ${ }^{43}$ When added to a program of AK removal by photodynamic therapy (PDT), photolyase in liposomes with sunscreen reduced the appearance of new AKs after PDT treatment in 15 patients compared to sunscreen alone. $^{44}$

These studies have focused on patients at risk for skin cancer, because of either genetic predisposition or history of pre-cancers, and they consistently show a reduction of
AKs and, in some cases, a reduction in basal cell carcinomas.

\section{Question 5: can these enzymes repair past damage?}

DNA repair mechanisms must act relatively shortly after induction of DNA damage, prior to them blocking transcription or causing errors in replication. This has led many to presume that DNA repair enhancement must occur hours after UV exposure to have any benefit, and that by middle age sun damage to skin is irreversible.

The data from both laboratory and clinical research demonstrate this is not true. As reviewed in Question 2, DNA damage initiates cellular responses, such as immune suppression, that facilitate the outgrowth of by-standing tumor cells. DNA repair should therefore preserve immune surveillance of the skin throughout our lifetime. From this, we would expect that increased DNA repair would:

- Act quickly within months to restore immunocompetence, rather than the years required for mutation to be expressed. In the first study of T4 endonuclease V administered to XP patients, the reduction in new AKs was observed within 3 months. ${ }^{37}$ Detailed examination of skin histology in $13 \mathrm{AK}$ patients after just 4 weeks of photolyase treatment showed improved skin parameters and biomarkers, such as reduced erythema, scaling, and atypical rounded nuclei in the spinosum and granular layers. ${ }^{45}$ Adding photolyase treatment to photodynamic therapy caused patients with a history of AKs to experience a suppression in the number of AKs starting 3 months after therapy, and continuing through 9 months post-therapy, compared to those patients treated with PDT alone. ${ }^{6}$

- Cause AKs to regress when the facilitating cellular responses were removed. Regression of AKs was in fact observed in a study of 50 patients with AKs using photolyase in a sunscreen compared to sunscreen alone. ${ }^{46}$ In another study of $11 \mathrm{AK}$ patients, treatment with photolyase in sunscreen led to a $75 \%$ reduction in lesion count within 3 months, ${ }^{47}$ and, in a third study, 9 patients achieved a 50\% reduction in $\mathrm{AK}$ counts within 3 months. ${ }^{43}$

- Suppress AKs and skin cancers even after discontinuing DNA repair enzyme treatment. In two studies, ${ }^{37,39}$ the rates of $\mathrm{AK}$ remained low even after discontinuing $\mathrm{T} 4$ endonuclease V or UV endonuclease repair enzyme 
treatment, suggesting that the enhanced repair removed an early signal in the tumorigenic process, and not simply suppressed a late step.

The most likely interpretation of these results is that repair of DNA damage today removes a necessary ingredient for the progression of past skin damage to pathological lesions.

\section{Question 6: how do DNA repair enzymes affect skin photoaging?}

While it is widely accepted that solar UV is responsible for photoaging, the precise role of DNA damage in accelerated aging of the skin is less clear. XP patients with defective DNA repair prematurely develop some characteristics of aging, such as surface freckling, lentigos and telangiectasias, but not others, such as deeper skin laxity, sagging and wrinkles. ${ }^{48}$ This may be explained by the extreme photosensitivity of the epidermal and vascular cells lying close to the surface and absorbing UVB, while deeper structural proteins are not as sensitive.

DNA repair enzymes applied to UV-exposed skin, however, do result in attenuation of cellular responses that are direct and indirect causes of photoaging. MMP-1 degrades the collagen support structure, a hallmark of photoaged skin, and is induced following UV exposure. Treatment of UV-exposed human cells with either T4 endonuclease $\mathrm{V}$ or UV endonuclease in liposomes reduced MMP-1 gene expression, and UV-irradiated human skin treated with photolyase in liposomes showed reduced MMP-1 enzyme expression. ${ }^{29}$ Telomere shortening is associated with premature senescence of cells, and treatment of human skin with photolyase in a sunscreen before UV exposure and UV endonuclease in liposomes afterward reduced telomere shortening compared to sunscreen alone and in fact prevented any measurable reduction. ${ }^{28}$

DNA repair enzymes do have a measurable effect on skin aging parameters. UV endonuclease, photolyase and OGG1 endonuclease in liposomes applied twice daily for 4 weeks to 32 subjects with fine lines in the eye area resulted in a significant reduction in crow's feet lines, and $72 \%$ of subjects noted improvement. ${ }^{49}$ Photolyase in a sunscreen applied to 50 patients with $\mathrm{AK}$ for 6 months resulted in a significant reduction in scaling, erythema and pigmentation compared to sunscreen alone. ${ }^{46}$

Although DNA damage may not be responsible for all signs of photoaging, DNA repair enhanced by enzyme treatment significantly improves several of its characteristics.

\section{Safety profile}

None of the reviewed studies reported adverse events specific to the proper use of the product nor did any express safety concerns. Many studies were silent on the point. The most extensive safety data have been reported for $\mathrm{T} 4$ endonuclease $\mathrm{V}$ in liposomes. ${ }^{21}$ Both acute and chronic safety testing in mice and humans showed neither adverse reactions nor significant changes in serum chemistry or in skin biology. Photolyase in sunscreen applied by 10 subjects twice daily for 6 months did not result in any adverse events. ${ }^{24}$ Photolyase in sunscreen applied by 17 patients with multiple AKs had no greater side effects (mainly erythema) than the group using sunscreen alone. ${ }^{46}$ A 9-month study of twice daily application of two different serums with DNA repair enzymes, as well as a sunscreen containing photolyase, among 15 patients in each arm found no adverse events. ${ }^{44}$ In a 1-year trial of photolyase in sunscreen by 8 patients with $\mathrm{XP}$, the investigators reported that the product was well tolerated and no relevant side effects were found. ${ }^{41}$ One study reported periorbital burning in one subject when the DNA repair product was accidentally contacted with the eye. ${ }^{39}$

\section{Summary}

Research over four decades and involving hundreds of patients and volunteers have investigated the use of DNA repair enzymes encapsulated in liposomes to mitigate sun damage to the skin. The mechanism by which the enzymes penetrate the skin, access damaged DNA and stimulate its repair has been outlined in cultured cells, mouse and human studies, but several details remain open questions. The safety profile is attractive because no serious side effects have been identified in any of the clinical trials.

DNA repair occurs in the context of dynamic cell metabolism, including time-sensitive transcription of potentially damaged DNA strands. The rate of removal of damage (prior to transcription or replication), and not the absolute level of repair, explains why enhanced repair has such a dramatic effect on cellular responses to UV. Prevention of AKs and skin cancer by enhanced DNA repair ha been reported in several clinical studies involving several hundred subjects. This is consistent with the prevailing model that DNA damage is converted to a mutation, which in rare instances alters a key oncogene and results in malignant transformation.

What has been surprising is the regression of preexisting AKs and measurable benefits observed in weeks and months, far shorter than might be predicted from the 
mutational theory of skin cancer. This suggests that sun damage to skin creates an environment that favors outgrowth of transformed cells, possible by impairing immune surveillance.

These observations suggest that enhancing DNA repair may improve signs of photoaging and may prevent skin cancers. The data is not yet conclusive, and this remains an open area for longer-term research to establish the role of DNA damage in skin aging and confirm its central role in initiation and promotion of skin cancer.

\section{Disclosure}

Daniel Yarosh consults for Pharma Cosmetics Inc. Ronald Moy is the scientific director and founder of DNA EGF Renewal and Cellular MD. The authors report no other conflicts of interest in this work.

\section{References}

1. US Department of Health and Human Services. The Surgeon General's Call to Action to Prevent Skin Cancer. Washington (DC): US Department of Health and Human Services, Office of the Surgeon General; 2014.

2. Zachos E, Rosen E. What sunscreens are best for you- and the planet? National Geographic Magazine. 21 May 2019. Available from: https:// www.nationalgeographic.com/travel/features/sunscreen-destroyingcoral-reefs-alternatives-travel-spd/. Accessed May 22, 2019.

3. Matta MK, Zusterzeel R, Pilli NR, et al. Effect of sunscreen application under maximal use conditions on plasma concentration of sunscreen active ingredients: a randomized clinical trial. JAMA. 2019. doi:10.1001/jama.2019.5586

4. FDA advances new proposed regulation to make sure that sunscreens are safe and effective. U.S. Food and Drug Administration. 21 February 2019. Available from: https://www.fda.gov/news-events/ press-announcements/fda-advances-new-proposed-regulation-makesure-sunscreens-are-safe-and-effective. Accessed May 22, 2019.

5. Yarosh D. 1991. United States Patent No. US 5077211. Available from: https://patents.google.com/patent/US5077211A/en. Accessed August 14, 2019

6. Bhatia N, Berman B, Ceilley RI, Kircik LH. Understanding the role of photolyases: photoprotection and beyond. $J$ Drugs Dermatol. 2017;16(5):61-66.

7. Cafardi JA, Elmets CA. T4 endonuclease V: review and application to dermatology. Expert Opin Biol Ther. 2008;8(6):829-838. doi:10.1517/14712598.8.6.829

8. Kabir Y, Seidel R, Mcknight B, Moy R. DNA repair enzymes: an important role in skin cancer prevention and reversal of photodamage-a review of the literature. J Drugs Dermatol. 2015;14(3):297303.

9. Megna M, Lembo S, Balato N, Monfrecola G. "Active" photoprotection: sunscreens with DNA repair enzymes. G Ital Dermatol Venereol. 2017;152(3):302-307. doi:10.23736/S0392-0488.17.0556 7-5

10. Rosenthal A, Stoddard M, Chipps L, Herrmann J. Skin cancer prevention: a review of current topical options complementary to sunscreens. J Eur Acad Dermatol Venereol. 2019. doi:10.1111/jdv,15522

11. Tanaka K, Okada Y, Sekiguchi M. Restoration of ultraviolet-induced unscheduled DNA synthesis of xeroderma pigmentosum cells by insertion of T4 endonuclease V utilizing HVJ. PNE. 1976;21 (7):525-535.
12. Kuraoka I. Diversity of endonuclease V: from DNA repair to RNA editing. Biomolecules. 2015;5(4):2194-2206. doi:10.3390/biom504 2194

13. Yarosh DB, Kibitel J, O'Connor A, Hejmadi V, Bennett P, Sutherland BM. DNA repair liposomes in antimutagenesis. J Env Path Tox Oncol. 1997;16(4):287-292.

14. Sutherland BM, Hausrath SG. Insertion of E. coli photoreactivating enzyme into V79 hamster cells. Nature. 1980;286(5772):510. doi: $10.1038 / 286510 \mathrm{a} 0$

15. Liu Z, Tan C, Guo X, et al. Dynamics and mechanism of cyclobutane pyrimidine dimer repair by DNA photolyase. Proc Natl Acad Sci. 2011;108(36):14831-14836. doi:10.1073/pnas.1110927108

16. Van Der Horst GT, Muijtjens M, Kobayashi K, et al. Mammalian Cry1 and Cry2 are essential for maintenance of circadian rhythms. Nature. 1999;398(6728):627. doi:10.1038/19323

17. Yarosh DB, Canning MT, Teicher D, Brown DA. After sun reversal of DNA damage: enhancing skin repair. Mutat Res. 2005;571(12):57-64. doi:10.1016/j.mrfmmm.2004.06.058

18. Yarosh DB. Liposomes in investigative dermatology. Photodermatol Photoimmunol Photomed. 2001;17(5):203-212.

19. Yarosh D, Bucana C, Cox P, Alas L, Kibitel J, Kripke M. Localization of liposomes containing a DNA repair enzyme in murine skin. J Invest Dermatol. 1994;103(4):461-468. doi:10.1111/15231747.ep12395551

20. Wolf P, Müllegger RR, Soyer HP, et al. Topical treatment with liposomes containing $\mathrm{T} 4$ endonuclease $\mathrm{V}$ protects human skin in vivo from ultraviolet-induced upregulation of interleukin-10 and tumor necrosis factor- $\alpha$. J Invest Dermatol. 2000;114(1):149-156. doi:10.1046/j.1523-1747.2000.00839.x

21. Yarosh D, Klein J, Kibitel J, et al. Enzyme therapy of xeroderma pigmentosum: safety and efficacy testing of T4N5 liposome lotion containing a prokaryotic DNA repair enzyme. Photoderm Photoimmunol Photomed. 1996;12(3):122-130. doi:10.1111/j.16000781.1996.tb00188.x

22. Ke MS, Camouse MM, Swain FR, et al. UV protective effects of DNA repair enzymes and RNA lotion. Photochem Photobiol. 2008;84(1):180-184. doi:10.1111/j.1751-1097.2007.00217.x

23. Stege H, Roza L, Vink AA, et al. Enzyme plus light therapy to repair DNA damage in ultraviolet-B-irradiated human skin. Proc Natl Acad Sci. 2000;97(4):1790-1795. doi:10.1073/pnas.030528897

24. Berardesca E, Bertona M, Altabas K, Altabas V, Emanuele E. Reduced ultraviolet-induced DNA damage and apoptosis in human skin with topical application of a photolyase-containing DNA repair enzyme cream: clues to skin cancer prevention. Mol Med Rept. 2012;5(2):570-574.

25. Carducci M, Pavone PS, De GM, et al. Comparative effects of sunscreens alone vs sunscreens plus DNA repair enzymes in patients with actinic keratosis: clinical and molecular findings from a 6month, randomized, clinical study. J Drugs Dermatol. 2015;14 (9):986-990.

26. Emanuele E, Spencer JM, Braun M. An experimental double-blind irradiation study of a novel topical product (TPF 50) compared to other topical products with DNA repair enzymes, antioxidants, and growth factors with sunscreens: implications for preventing skin aging and cancer. J Drugs Dermatol. 2014;13(3):309-314.

27. Vidal-Asensi S, Gutiérrez-Ortega C, Deagustín-Vázquez D, UghelliYampey G. Photolyase sunscreen decreases expression of p53. $J$ Amer Aad Dermatol. 2012;66(4).

28. Emanuele E, Altabas V, Altabas K, Berardesca E. Topical application of preparations containing DNA repair enzymes prevents ultravioletinduced telomere shortening and c-FOS proto-oncogene hyperexpression in human skin: an experimental pilot study. J Drugs Dermatol. 2013;12(9):1017-1021.

29. Dong KK, Damaghi N, Picart SD, et al. UV-induced DNA damage initiates release of MMP-1 in human skin. Exp Dermatol. 2008;17 (12):1037-1044. doi:10.1111/j.1600-0625.2008.00747.x 
30. Kuchel JM, Barnetson RS, Halliday GM. Cyclobutane pyrimidine dimer formation is a molecular trigger for solar-simulated ultraviolet radiation-induced suppression of memory immunity in humans. Photochem Photobiol Sci. 2005;4(8):577-582. doi:10.1039/b504068j

31. Lucas CR, Ke MS, Matsui MS, et al. Immune protective effect of a moisturizer with DNA repair ingredients. J Coms Dermatol. 2008;7 (2):132-135.

32. Hofer A, Legat FJ, Gruber-Wackernagel A, Quehenberger F, Wolf P. Topical liposomal DNA-repair enzymes in polymorphic light eruption. Photochem Photobiol Sci. 2011;10(7):1118-1128. doi:10.1039/ c1pp05009e

33. Yarosh D, Alas LG, Yee V, et al. Pyrimidine dimer removal enhanced by DNA repair liposomes reduces the incidence of UV skin cancer in mice. Cancer Res. 1992;52(15):4227-4231.

34. Bito T, Ueda M, Nagano T, Fujii S, Ichihashi M. Reduction of ultraviolet-induced skin cancer in mice by topical application of DNA excision repair enzymes. Photoderm Photoimmunol Photomed. 1995;11(1):913. doi:10.1111/j.1600-0781.1995.tb00130.x

35. Sha Y, Vartanian V, Owen N, et al. Modulation of UVB-induced carcinogenesis by activation of alternative DNA repair pathways. Sci Rep. 2018;8(1):705. doi:10.1038/s41598-017-17940-8

36. Wulff BC, Schick JS, Thomas-Ahner JM, Kusewitt DF, Yarosh DB, Oberyszyn TM. Topical treatment with OGG1 enzyme affects UVBinduced skin carcinogenesis. Photochem Photobiol. 2008;84(2):317321. doi:10.1111/j.1751-1097.2007.00257.x

37. Yarosh D, Klein J, O'Connor A, Hawk J, Rafal E, Wolf P; Xeroderma Pigmentosum Study Group. Effect of topically applied T4 endonuclease V in liposomes on skin cancer in xeroderma pigmentosum: a randomised study. Lancet. 2001;357(9260):926-929. doi:10.1016/s0140-6736(00)04214-8

38. DeBoyes T, Kouba D, Ozog D, et al. Reduced number of actinic keratoses with topical application of DNA repair enzyme creams. $J$ Drugs Dermol. 2010;9(12):1519-1521.

39. Stoddard M, Herrmann J, Moy L, Moy R. Improvement of actinic keratoses using topical DNA repair enzymes: a randomized placebocontrolled trial. J Drugs Dermatol. 2017;16(10):1030-1034.

40. Puig S, Granger C, Garre A, Trullàs C, Sanmartin O, Argenziano G. Review of clinical evidence over 10 years on prevention and treatment of a film-forming medical device containing photolyase in the management of field cancerization in actinic keratosis. Dermatol Ther. 2019;9(2):259-270. doi:10.1007/s13555-019-0294-1
41. Giustini S, Miraglia E, Berardesca E, Milani M, Calvieri S. Preventive long-term effects of a topical film-forming medical device with ultra-high UV protection filters and DNA repair enzyme in xeroderma pigmentosum: a retrospective study of eight cases. Case Rep Dermatol. 2014;6(3):222-226. doi:10.1159/ 000368182

42. Puviani M, Barcella A, Milani M. Efficacy of a photolyase-based device in the treatment of cancerization field in patients with actinic keratosis and non-melanoma skin cancer. G Ital Dermatol Venereol. 2013;148(6):693-698.

43. Navarrete-Dechent C, Molgó M. The use of a sunscreen containing DNA-photolyase in the treatment of patients with field cancerization and multiple actinic keratoses: a case-series. Dermatol Online J. 2017;23(1).

44. Eibenschutz L, Silipo V, De Simone P, et al. A 9-month, randomized, assessor-blinded, parallel-group study to evaluate clinical effects of film-forming medical devices containing photolyase and sun filters in the treatment of field cancerization compared with sunscreen in patients after successful photodynamic therapy for actinic keratosis. Br J Dermatol. 2016;175(6):1391.

45. Puig S, Puig-Butillé JA, Díaz MA, Trullas C, Malvehy J. Field cancerisation improvement with topical application of a film-forming medical device containing photolyase and UV filters in patients with actinic keratosis, a pilot study. J Clin Exp Dermatol Res. 2014;5:3.

46. Moscarella E, Argenziano G, Longo C, Aladren S. Management of cancerization field with a medical device containing photolyase: a randomized, double-blind, parallel-group pilot study. $J$ Eur Acad Dermatol Venereol. 2017;31:e401-e403. doi:10.1111/ jdv. 14326

47. Puviani M, Milani M. A pilot, prospective, open-label study on the effects of a topical photorepair and photoprotection film-forming medical device in patients with actinic keratoses evaluated by means of skin analysis camera antera 3D. J Clin Exp Dermatol Res. 2015;6(263):2.

48. DiGiovanna JJ, Kraemer KH. Shining a light on xeroderma pigmentosum. J Invest Dermatol. 2012;132(3):785-796. doi:10.1038/ jid. 2011.426

49. Walfield AM, Yarosh DB. It's never too late: DNA repair and photoaging. Cosm Toil. 2007;122(7).

\section{Publish your work in this journal}

Clinical, Cosmetic and Investigational Dermatology is an international, peer-reviewed, open access, online journal that focuses on the latest clinical and experimental research in all aspects of skin disease and cosmetic interventions. This journal is indexed on CAS.
The manuscript management system is completely online and includes a very quick and fair peer-review system, which is all easy to use. Visit http://www.dovepress.com/testimonials.php to read real quotes from published authors. 